\title{
Influência do periodo de abstinência sexual sobre as características seminais de homens inférteis
}

\author{
Influence of abstinence period on seminal characteristics in infertile men
}

Fábio Firmbach Pasqualotto ${ }^{1}$, Gabriela Poglia Fonseca ${ }^{2}$, Matheus Luís da Silva ${ }^{2}$, Ramon Venzon Ferreira ${ }^{2}$, Bibiana Elisa Zago ${ }^{3}$, Cláudio Garbin Júnior ${ }^{3}$, Eleonora Bedin Pasqualotto ${ }^{4}$

\section{RESUMO}

Objetivo: avaliar a influência do período de abstinência sexual sobre os parâmetros espermáticos em homens inférteis, assim como determinar a melhora na qualidade seminal após mistura dos ejaculados. Métodos: estudo retrospectivo no qual se avaliou um grupo de 88 homens com oligozoospermia $(n=25)$, astenozoospermia $(n=43)$ ou oligoastenozoospermia $(n=20)$, cujas parceiras foram submetidas à inseminação intra-uterina entre setembro de 2002 e dezembro de 2004. Foram excluídos da análise casais nos quais os homens apresentavam análise seminal normal ou mulheres com alterações sugestivas de infertilidade. Cada homem produziu duas amostras seminais em período curto de tempo (30 minutos a 1 hora). Foram avaliados o volume seminal, a concentração total de espermatozóides móveis e o percentual de motilidade espermática. Para todos os parâmetros avaliados foram comparadas a primeira e a segunda amostra seminal coletadas. Além disso, a concentração total de espermatozóides móveis foi comparada entre a primeira amostra coletada e a união das amostras. A avaliação estatística foi realizada usando o teste t e o teste $\chi^{2}$. Resultados: em homens oligozoospérmicos não houve diferença nas características seminais entre a primeira e a segunda amostra seminal ( $p>0,05)$. A concentração total de espermatozóides móveis aumentou significativamente na segunda amostra em comparação à primeira amostra em homens astenozoospérmicos $\left(42,4 \pm 6,8\right.$ vs $51,5 \pm 7,2 \times 10^{6}$ espermatozóides/mL) e oligoastenozoospérmicos $\left(11,1 \pm 7,4 v s\right.$ 14,35 $\pm 7,2 \times 10^{6}$ espermatozóides $/ \mathrm{mL}(\mathrm{p}<0,05)$. A união dos dois ejaculados aumentou a concentração total de espermatozóides móveis em comparação à primeira amostra $(\mathrm{p}<0,05)$ nos homens oligozoospérmicos, astenozoospérmicos e oligoastenozoospérmicos em 110,5, 110,3 e 136,03\%, respectivamente. Conclusões: menor período de abstinência está associado a melhora da motilidade em homens inférteis. A união das amostras seminais constitue alternativa para aumentar a concentração total de espermatozóides móveis neste grupo de pacientes que desejam participar de inseminação intra-uterina ao invés da fertilização in vitro.

PALAVRAS-CHAVE: Abstinência sexual; Infertilidade masculina; Sêmen; Oligoespermia; Espermatozóides

\section{ABSTRACT}

Purpose: to evaluate the influence of abstinence period on seminal characteristics in infertile men and to establish a better seminal quality after pooling the samples. Methods: a retrospective study was performed on 88 oligozoospermic $(\mathrm{n}=25)$, asthenozoospermic $(n=43)$, and oligoasthenozoospermic $(n=20)$ men whose partners underwent intrauterine insemination between September 2002 and December 2004. We excluded men with a normal semen analysis or women with abnormalities suggestive of infertility. Each man produced two semen samples in a short period of time ( $30 \mathrm{~min}$ to $1 \mathrm{~h}$ ). We evaluated semen volume, total motile sperm count and percentage sperm motility. Comparisons were made between the first and second semen samples. After pooling the samples, we compared the total motile sperm count between the first sample and the pooled samples. Statistical evaluation was performed by Student's $t$ test and the $\chi^{2}$ test. Results: in oligozoospermic men, there were no differences in the semen characteristics between the first and the second seminal samples $(\mathrm{p}>0.05)$. The total motile sperm count increased significantly in the second sample in comparison to the first sample in asthenozoospermic $\left(42.4 \pm 6.8 v s 51.5 \pm 7.2 \times 10^{6} \mathrm{sperm} / \mathrm{mL}\right)$ and oligoasthenozoospermic men $\left(11.1 \pm 7.4 v s 14.35 \pm 7.2 \times 10^{6} \mathrm{sperm} / \mathrm{mL}(\mathrm{p}<0.05)\right.$. The

Centro de Ciências Biológicas e da Saúde, Universidade de Caxias do Sul - UCS - Caxias do Sul (RS), Brasil

1 Professor Doutor Titular de Urologia e Embriologia da Faculdade de Medicina da Universidade de Caxias do Sul - UCS - Caxias do Sul (RS), Brasil.

2 Acadêmicos do Curso de Medicina da Universidade de Caxias do Sul - UCS - Caxias do Sul (RS), Brasil.

3 Acadêmicos do Curso de Medicina da Universidade de Caxias do Sul - UCS - Caxias do Sul (RS), Brasil.

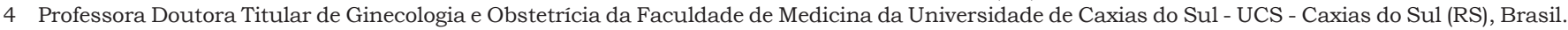

Correspondência: Fabio Firmbach Pasqualotto

Rua Pinheiro Machado, 2569, sl 23/24 - Bairro São Pelegrino - 95020-172 - Caxias do Sul - RS - e-mail: Fabio@conception-rs.com.br 
pool of two ejaculates increased the total motile sperm count in comparison to the first sample $(p<0.05)$ in oligozoospermic, asthenozoospermic and oligoasthenozoospermic men by $110.5,110.3$ and $136.03 \%$, respectively. Conclusions: a short period of abstinence is associated with higher sperm motility in infertile men. The pool of two semen samples is a way to increase the total motile sperm count in this group of patients whose wives want to undergo an intrauterine insemination instead of in vitro fertilization.

KEYWORDS: Sexual abstinence; Infertility, male; Semen; Oligospermia; Spermatozoa

\section{Introdução}

Na maioria dos países desenvolvidos, a média de idade dos pais tem aumentado e uma grande parcela de homens tem iniciado a sua prole após os 50 anos de idade ${ }^{1}$. Esta tendência deve-se ao fato de que as mulheres estão demorando muito para tomar a decisão de ter o primeiro filho em idade na qual já inicia o declínio de sua fertilidade. Apesar de ainda não estar bem definida a influência da idade na qualidade seminal, estudo recente demonstrou que existe piora na concentração e na morfologia espermática após os 45 anos de idade ${ }^{2}$.

A variação na qualidade seminal em face fatores individuais e ambientais já foi bem estabelecida pela experiência clínica e por diversos estudos, sendo o período de abstinência sexual um dos fatores responsáveis por mudanças nas características seminais ${ }^{3,4}$. Estudo realizado na Dinamarca em 2004 demonstrou existir aumento de $25,2 \%$ ao dia na concentração espermática e de $9,5 \%$ ao dia no volume seminal nos primeiros quatro dias de abstinência ${ }^{3}$. Além disso, não houve alteração na motilidade e morfologia dos espermatozóides com relação à duração da abstinência. Já outros autores observaram importante decréscimo da motilidade com o aumento do período de abstinência após 5 dias ${ }^{5,6}$.

$\mathrm{Na}$ tentativa de minimizar esta variação, a maioria dos profissionais seguem as diretrizes estabelecidas pela Organização Mundial de Saúde (OMS), que recomenda um período de abstinência entre dois e cinco dias para a avaliação dos parâmetros seminais. Porém, estas recomendações não são baseadas em evidências sólidas na literatura para comprovar sua relevância estatísti$\mathrm{ca}^{7}$.

Um grande número de tratamentos para infertilidade está disponivel atualmente, entre estes a inseminação intra-uterina (IIU) com ou sem indução da ovulação e a fertilização in vitro (FIV) com ou sem micromanipulação. A qualidade seminal tem grande importância para a escolha da técnica de reprodução assistida a ser utilizada ${ }^{8}$. Pacientes com concentração espermática inferior a $5 \times 10^{6}$ espermatozóides $/ \mathrm{mL}$ após processamento seminal têm indicação de FIV, já que pacientes com estes parâmetros seminais possuem baixas taxas de gravidez com outros métodos de tratamento para infertilidade 9 . Porém, o tratamento de escolha em pacientes que apresentem fator masculino leve a moderado é a IIU ${ }^{10}$, método este mais simples e econômico que a FIV. Em virtude do fato de que na maioria dos hospitais brasileiros não se realizam procedimentos de FIV gratuitos para a população, é importante realizarmos tratamentos que resultem em diminuição dos gastos a serem feitos pelos pacientes.

O objetivo deste estudo foi avaliar os parâmetros espermáticos em homens inférteis ao diminuir o período de abstinência entre duas amostras seminais e ao se juntarem estes ejaculados.

\section{Métodos}

Este estudo prospectivo foi aprovado pelo Comitê de Ética e Pesquisa da Universidade de Caxias do Sul e todos os participantes do estudo assinaram termo de consentimento informado. No período de setembro de 2002 a dezembro de 2004, 326 homens procuraram o serviço para investigação da infertilidade masculina. Destes 326 homens, 218 apresentaram alteração seminal (61 com oligozoospermia, 43 com astenozoospermia, 53 com teratozoospermia, 41 com oligoastenozoospermia e 20 com oligoastenoteratozoospermia). Destes, 88 casais cujos homens apresentavam oligozoospermia $(n=25)$, astenozoospermia $(n=43)$ ou oligoastenozoospermia $(n=20)$ foram submetidos ao tratamento de infertilidade conjugal por meio da inseminação intra-uterina e foram incluídos no estudo. Os pacientes com análise seminal normal (108 homens) e aqueles pacientes que foram submetidos à FIV ou que não desejaram realizar tratamento com IIU foram excluídos do estudo. Além disso, as mulheres com alterações sugestivas de infertilidade feminina foram, também, excluídas do estudo.

Os pacientes foram encaminhados para investigação de infertilidade por ginecologistas, 
urologistas ou procura espontânea. A avaliação dos pacientes foi realizada por um urologista especialista em infertilidade masculina (FFP). Todas as parceiras foram investigadas quanto à infertilidade feminina por uma ginecologista especialista em reprodução humana (EBP) por meio do exame físico, exames hormonais, ultra-sonografia e histerossalpingografia, não sendo detectadas alterações.

Os 88 pacientes foram orientados a coletar suas amostras seminais por masturbação dentro de recipiente plástico estéril. As amostras foram submetidas à liquefação a $37^{\circ} \mathrm{C}$ por 30 minutos, sendo avaliados os seguintes parâmetros seminais: volume seminal, percentual de motilidade espermática e concentração total de espermatozóides móveis. O volume do ejaculado foi medido por aspiração de toda a amostra com o auxílio de uma pipeta graduada acoplada a um pipetador eletrônico. Após misturar cuidadosamente a amostra seminal por completo, com o auxílio de aparelho misturador de amostras, as mesmas foram avaliadas manualmente pelo pesquisador. Para cada mensuração, uma alíquota liquefeita de $5 \mu \mathrm{L}$ foi inserida em uma câmara de contagem Microcell, descartável e com $20 \mu \mathrm{m}$ de profundidade, até seu preenchimento integral, com o auxílio de pipeta de pressão positiva para determinação da concentração e motilidade espermáticas. Estas foram analisadas manualmente com o uso de microscópio óptico equipado com objetiva de contraste de fase de 20X e aumento de 200X.

No dia da IIU, cada homem produziu duas amostras seminais em um período curto de tempo (entre 30 minutos e uma hora). Comparações foram feitas entre a primeira e a segunda amostra coletada, assim como o percentual de melhora na concentração total de espermatozóides móveis após se juntarem as duas amostras coletadas com relação à primeira amostra seminal.

As parceiras dos pacientes foram submetidas à indução da ovulação com hormônio folículoestimulante recombinante (FSHr) diariamente na dose inicial de 50 a 100 UI/dia, subcutâneo, iniciando-se no segundo ou terceiro dia do ciclo menstrual. A monitorização da indução da ovulação foi feita pela ultra-sonografia transvaginal seriada com transdutor de $5 \mathrm{MHz}$, sendo o primeiro exame realizado antes de iniciar o estímulo (ultra-sonografia basal) e o segundo no quinto dia de estímulo. A partir do quinto dia de estímulo, o controle ultra-sonográfico foi realizado a cada dois dias e a dose de gonadotrofina administrada foi ajustada de acordo com a medida e quantidade dos folículos. Quando pelo menos um folículo atingiu $20 \mathrm{~mm}$ de diâmetro médio, cinco mil unidades de gonadotrofina coriônica humana (hCG) foram administradas por via intramuscular. A IIU foi realizada 36 horas após a injeção de hCG. O ciclo foi cancelado quando a ultra-sonografia revelou, no momento da administração do hCG, um número de cinco ou mais folículos com diâmetro superior a $16 \mathrm{~mm}$, para evitar gestação múltipla e os riscos inerentes a esta. O diagnóstico da gestação foi realizado por exame plasmático de beta-hCG a partir de 14 dias da realização da IIU, em casos nos quais não ocorreu a menstruação.

O teste $t$ de Student e o teste do $\chi^{2}$ foram utilizados para comparar as médias de motilidade, concentração total de espermatozóides móveis e volume seminal entre a primeira e segunda coleta no mesmo paciente. O teste $t$ de Student (dados paramétricos) foi utilizado para comparar as médias das variáveis examinadas e o teste do $\chi^{2}$ para comparar as taxas percentuais de aumento nas variáveis examinadas. O nível de diferença estatística estabelecido foi 5\%. A informação foi analisada pelo programa Statistical Package for Social Sciences, SPPS, versão 11.0 (SPSS Inc., Chicago, IL, EUA).

\section{Resultados}

Em homens oligozoospérmicos, as características seminais da primeira e da segunda amostras foram similares $\left(12,4 \pm 3,5\right.$ vs. $13,5 \pm 3,1 \times 10^{6} \pm 3,1$ espermatozóides $/ \mathrm{mL} ; \mathrm{p}=0,13$ ) (Tabela 1 ). Da mesma maneira, detectamos leve diminuição no volume seminal $(2,9 \pm 0,8$ e $2,6 \pm 0,9 \mathrm{~mL} ; \mathrm{p}=0,17)$ e aumento na concentração de espermatozóides móveis na segunda amostra, apesar de não se alcançar diferença significante $(7,6 \pm 3,9$ vs $8,9 \pm 3,6 \times 10^{6}$ espermatozóides $\left./ \mathrm{mL} ; \mathrm{p}=0,08\right)$ Além disso, não houve melhora na motilidade espermática $(57,4 \pm 6,5$ e $64,9 \pm 8,5 \%$ de espermatozóides móveis; $\mathrm{p}=0,07)$.

Tabela 1 - Variáveis relacionadas à qualidade seminal analisadas em homens oligozoospérmicos.

\begin{tabular}{|c|c|c|c|}
\hline Variáveis & $\begin{array}{l}\text { Primeira } \\
\text { amostra }\end{array}$ & $\begin{array}{l}\text { Segunda } \\
\text { amostra }\end{array}$ & $\begin{array}{c}\text { Valor de } \\
\mathrm{p}\end{array}$ \\
\hline Volume seminal (mL) & $2,9 \pm 0,8$ & $2,6 \pm 0,9$ & 0,17 \\
\hline $\begin{array}{l}\text { Concentração } \\
\text { (x106 espermatozóides } / \mathrm{mL})\end{array}$ & $12,4 \pm 3,5$ & $13,5 \pm 3,1$ & 0,13 \\
\hline Motilidade (\%) & $57,4 \pm 6,5$ & $64,9 \pm 8,5$ & 0,07 \\
\hline $\begin{array}{l}\text { Concentração total de } \\
\text { espermatozóides móveis } \\
\text { (x106 espermatozóides } / \mathrm{mL})\end{array}$ & $7,6 \pm 3,9$ & $8,9 \pm 3,6$ & 0,08 \\
\hline
\end{tabular}


Em homens astenozoospérmicos $(n=43)$, a motilidade e a concentração total de espermatozóides móveis aumentaram significativamente $(\mathrm{p}<0,01)$ na segunda amostra $(34,8 \pm 5,6$ vs $47,3 \pm 6,1 \%$ e $42,4 \pm 6,8$ vs $51,5 \pm 7,2 \times 10^{6}$ espermatozóides $/ \mathrm{mL}$ ); respectivamente (Tabela 2). Decréscimo no volume seminal, porém não significativo, também foi detectado neste grupo $(2,7 \pm 0,7 \mathrm{e}$ $2,5 \pm 0,6 \mathrm{~mL} ; \mathrm{p}=0,21)$.

Tabela 2 - Variáveis relacionadas à qualidade seminal analisadas em homens astenozoospérmicos.

\begin{tabular}{lccc}
\hline Variáveis & $\begin{array}{r}\text { Primeira } \\
\text { amostra }\end{array}$ & $\begin{array}{c}\text { Segunda } \\
\text { amostra }\end{array}$ & $\begin{array}{c}\text { Valor de } \\
\mathbf{p}\end{array}$ \\
\hline Volume seminal $(\mathrm{mL})$ & $2,7 \pm 0,7$ & $2,5 \pm 0,6$ & 0,21 \\
$\begin{array}{l}\text { Concentração } \\
\text { (x106 espermatozóides } / \mathrm{mL})\end{array}$ & $36,85 \pm 12,4$ & $42,55 \pm 9,7$ & 0,06 \\
Motilidade (\%) & $34,8 \pm 5,6$ & $47,3 \pm 6,1$ & 0,03 \\
Concentração total de & $42,4 \pm 6,8$ & $51,5 \pm 7,2$ & 0,04 \\
$\quad \begin{array}{l}\text { espermatozóides móveis } \\
\text { (x106 espermatozóides } / \mathrm{mL})\end{array}$ & & & \\
\hline
\end{tabular}

$p<0,05$ foi considerado estatisticamente significativo.

A motilidade e a concentração total de espermatozóides móveis aumentaram significativamente $(\mathrm{p}<0,01)$ na segunda amostra $(32,3 \pm 4,2$ vs. $44,3 \pm 7,2 \%$ e $11,1 \pm 7,4$ vs $14,35 \pm 7,2 \times 10^{6}$ espermatozóides $/ \mathrm{mL}$ ), respectivamente, no grupo de pacientes oligoastenozoospérmicos $(n=20)(T a-$ bela 3).

Tabela 3 - Variáveis relacionadas à qualidade seminal analisadas em homens oligoastenozoospérmicos.

\begin{tabular}{|c|c|c|c|}
\hline Variáveis & $\begin{array}{l}\text { Primeira } \\
\text { amostra }\end{array}$ & $\begin{array}{l}\text { Segunda } \\
\text { amostra }\end{array}$ & $\begin{array}{l}\text { Valor de } \\
\qquad p\end{array}$ \\
\hline Volume seminal (mL) & $2,8 \pm 0,5$ & $2,7 \pm 0,8$ & 0,09 \\
\hline $\begin{array}{l}\text { Concentração } \\
\text { (x10 espermatozóides } / \mathrm{mL})\end{array}$ & $9,01 \pm 2,1$ & $15,6 \pm 2,5$ & 0,06 \\
\hline Motilidade (\%) & $32,3 \pm 4,2$ & $44,3 \% \pm 7,2$ & 0,04 \\
\hline $\begin{array}{l}\text { Concentração total de } \\
\text { espermatozóides móveis } \\
\text { (x106 espermatozóides } / \mathrm{mL})\end{array}$ & $11,1 \pm 7,4$ & $14,35 \pm 7,2$ & 0,03 \\
\hline
\end{tabular}

$p<0,05$ foi considerado estatisticamente significativo.

A união dos dois ejaculados aumentou a concentração total de espermatozóides móveis em comparação à primeira amostra $(\mathrm{p}<0,05)$ nos homens oligozoospérmicos, astenozoospérmicos e oligoastenozoospérmicos em 110,5, 110,3 e $136,03 \%$, respectivamente (Tabela 4).
Tabela 4 - Concentração total de espermatozóides móveis da primeira amostra e após a mistura dos ejaculados.

\begin{tabular}{lcccc}
\hline $\begin{array}{l}\text { Parâmetros } \\
\text { seminais }\end{array}$ & $\begin{array}{r}\text { Primeira } \\
\text { amostra }\end{array}$ & $\begin{array}{l}\text { União dos } \\
\text { ejaculados }\end{array}$ & $\begin{array}{c}\text { Porcentagem } \\
\text { de melhora } \\
(\%)\end{array}$ & $\begin{array}{c}\text { Valor } \\
\text { de }\end{array}$ \\
\hline Oligozoospermia & $7,6 \pm 3,9$ & $16,1 \pm 1,2$ & 110,5 & 0,03 \\
Astenozoospermia & $42,4 \pm 6,8$ & $89,2 \pm 8,1$ & 110,3 & 0,03 \\
Oligoastenozoospermia & $11,1 \pm 7,4$ & $26,2 \pm 8,6$ & 136,03 & 0,02 \\
\hline
\end{tabular}

$p<0,05$ foi considerado estatisticamente significativo.

\section{Discussão}

Nas últimas décadas, foi identificado declínio na qualidade do sêmen de homens normais. Uma análise de 61 estudos revelou declínio na concentração espermática e no volume seminal no período entre 1938 e $1990^{11}$. Outro estudo também demonstrou menor concentração espermática assim como redução na motilidade e no número de espermatozóides morfologicamente normais entre 1973 e 1992 em homens férteis ${ }^{5}$. Embora a idade masculina afete a probabilidade de engravidar suas parceiras, anormalidades nos cromossomos dos espermatozóides e em alguns componentes da análise seminal são de importância inferior quando comparadas à freqüência das ejaculações ${ }^{1}$.

Da mesma maneira, o número de mulheres inférteis aumentou nos últimos 20 anos diante do adiamento na constituição familiar e concepção, levando a maior procura por tratamentos para infertilidade $^{12}$. De fato, houve grande tendência nestes últimos anos de as mulheres terem o seu primeiro filho quando a capacidade reprodutiva começa a diminuir ${ }^{1}$. Esta tendência estimulou o interesse de estudiosos a pesquisar os fatores associados ao declínio na fertilidade feminina. Atualmente sabemos que a qualidade oocitária diminui, ao menos parcialmente, devido ao aumento nas taxas de aneuploidia. O declínio da fertilidade tornase clinicamente relevante quando a mulher chega aos 35 anos de idade, e mesmo os tratamentos de reprodução assistida não conseguem compensar este declínio causado pela idade.

Vários fatores mostram influência nos parâmetros seminais como fumo, café, agrotóxicos e período de abstinência sexual ${ }^{6}$. Com relação ao periodo de abstinência sexual, são observadas diferenças importantes entre populações de pacientes férteis ou inférteis ${ }^{13,14}$. Estudo recente demonstra que periodos de abstinência sexual superiores a 3 dias diminuem as chances de um casal conseguir engravidar por meio de inseminação intrauterina, provavelmente decorrente da perda da 
viabilidade espermática ou por fatores não observados na análise seminal de rotina ${ }^{15}$. Avaliando o sêmen de homens normozoospérmicos, observase que, com o aumento do período de abstinência, ocorre incremento na concentração espermática e no volume seminal, porém a qualidade espermática sofre deterioração, havendo diminuição da motilidade e do número de espermatozóides morfologicamente normais ${ }^{3,5,6,8,16,17}$.

Por outro lado, em pacientes oligozoospérmicos, os parâmetros seminais sofrem diminuição da motilidade e morfologia espermática com o aumento da duração da abstinência ${ }^{6,17}$. Evitar abstinência sexual ou realizar abstinência de apenas um dia em homens oligozospérmicos é recomendável com o objetivo de alcançar a melhor qualidade seminal, visto que a morfologia espermática apresenta piora após o primeiro dia de abstinência sexual ${ }^{6}$.

Acredita-se que a motilidade espermática seja um dos parâmetros seminais com maior impacto sobre a fertilidade, sendo que a baixa motilidade está relacionada com maior dificuldade de concepção tanto espontaneamente como com o auxílio da inseminação intra-uterina ${ }^{6,16}$. Várias explicações fisiopatológicas foram elaboradas para esclarecer as diferenças entre populações férteis e inférteis em face do periodo de abstinência. A primeira justificativa para estes achados foi a importância do epidídimo e das glândulas sexuais acessórias influenciando o status funcional dos gametas masculinos ${ }^{6,18,19}$. É de conhecimento geral que, após a espermatogênese, ocorre uma fase de maturação dos espermatozóides na cauda do epidídimo onde estes permanecem imóveis até o momento da ejaculação ${ }^{20}$. $\mathrm{O}$ trânsito pelo epidídimo é influenciado por fatores externos como o estímulo sexual e a freqüência ejaculatória, os quais aumentam a velocidade da passagem dos espermatozóides. A motilidade então é induzida quando o ejaculado se junta às secreções das glândulas sexuais acessórias ${ }^{6}$.

A diminuição das taxas de produção espermática não reduz o número de espermatozóides no epidídimo ${ }^{21}$. Porém, o tempo de transporte dos espermatozóides no epidídimo é três vezes maior em oligozoospérmicos quando comparados aos normozoospérmicos, levando a deterioração da qualidade seminal. A senescência espermática causa decréscimo no percentual de espermatozóides morfologicamente normais, havendo, conseqüentemente, prejuízo na motilidade total. Este fenômeno é mais evidente quanto maior for o período de abstinência ${ }^{6,22}$.

Um aumento nos níveis de espécies reativas de oxigênio em pacientes inférteis foi encontrado ao se compararem as amostras seminais de homens férteis e de pacientes azoospérmicos ${ }^{23}$. Esta pode ser uma das razões para a rápida deterioração da motilidade e morfologia espermática em um periodo de abstinência mais prolongado nestes pacientes. A qualidade seminal sofre piora importante com o aumento nos niveis de espécies reativas de oxigênio no sêmen ${ }^{24,25}$.

Em estudo realizado em Israel, amostras seminais de ejaculados seqüenciais foram reunidas, observando-se resultados surpreendentes: a motilidade aumentou 49\% com relação à primeira amostra em normozoospérmicos, 95\% em astenozoospérmicos, 67 e $75 \%$ em oligozoospérmicos e 233 e $139 \%$ em oligoastenozoospérmicos ${ }^{26}$. Desta forma, usando metodologia semelhante à nossa, os autores mostraram também aumento na qualidade seminal do segundo ejaculado comparado ao primeiro. Outro estudo, no qual se compararam amostras seminais de pacientes oligozoospérmicos com intervalo de uma a quatro horas, demonstrou aumento na concentração total de espermatozóides móveis na segunda amostra em comparação à primeira ${ }^{9}$. Os mesmo autores demonstraram melhora de 329\% na concentração total de espermatozóides móveis quando foram unidas as duas amostras seminais com intervalo de até quatro horas comparado à primeira amostra seminal.

Os resultados deste estudo são de extrema importância para serem utilizados em casais nos quais o fator masculino é o causador da infertilidade. Homens oligozoospérmicos podem aumentar suas chances de fertilidade tendo relação sexual todos os dias no período ovulatório, assim como a união dos ejaculados se mostra um método simples e com um custo mais acessivel a fim de aumentar a motilidade total para inseminação intrauterina, entre outros tratamentos de reprodução assistida.

\section{Referências}

1. Baird DT, Collins J, Egozcue J, Eyers LH, Gianaroli L, Leridon H, et al. Fertility and ageing. Hum Reprod Update. 2005;11(3):261-76.

2. Pasqualotto FF, Sobreiro BP, Hallak J, Pasqualotto EB, Lucon AM. Sperm concentration and normal sperm morphology decrease and follicle-stimulating homone level increases with age. BJU Int. 2005;96(7):1087-91.

3. Carlsen E, Petersen JH, Andersson AM, Skakkebaek NE. Effects of ejaculatory frequency and season on variations in semen quality. Fertil Steril. 2004;82(2):358-66. 
4. Makkar G, Ng EH, Yeung WS, Ho PC. A comparative study of raw and prepared semen samples from two consecutive days. J Reprod Med. 2001;46(6):565-72.

5. Auger J, Kunstmann JM, Czyglik F, Jouannet P. Decline in semen quality among fertile men in Paris during the past 20 years. N Engl J Med. 1995;332(5):281-5.

6. Elzanazy S, Malm J, Giwercman A. Duration of sexual abstinence: epididymal and accessory sex gland secretions and their relationship to sperm motility. Hum Reprod. 2005;20(1):221-25.

7. Pasqualotto FF, Sobreiro BP, Hallak J, Athayde KS, Pasqualotto EB, Lucon AM. High percentage of abnormal semen parameters in a pre-vasectomy population. Fertil Steril. In press 2006.

8. Levitas E, Lunenfeld E, Weiss N, Friger M, Har-Vardi I, Koifman A, et al. Relationship between the duration of sexual abstinence and semen quality: analysis of 9,489 semen samples. Fertil Steril. 2005;83(6):1680-6.

9. Tur-Kaspa I, Dudkiewicz A, Confino E, Gleicher N. Pooled sequential ejaculates: a way to increase the total number of motile sperm from oligozoospermic men. Fertil Steril. 1990;54(5):906-9.

10. Garceau L, Henderson J, Davis LJ, Petrou S, Henderson LR, McVeigh E, et al. Economic implications of assisted reproductive techniques: a systematic review. Hum Reprod. 2002;17(12):3090109.

11. Carlsen E, Giwercman A, Keiding N, Skakkebaek NE. Evidence for decreasing quality of semen during past 50 years. BMJ. 1992;305(6854):609-13.

12. Stephen EH, Chandra A. Update projection of infertility in the United States: 1995-2025. Fertil Steril. 1998;70(1):30-4.

13. De Jonge C, LaFromboise M, Bosmans E, Ombelet W, Cox A, Nijs M. Influence of the abstinence period on human sperm quality. Fertil Steril. 2004;82(1):5765.

14. Kunzle R, Mueller MD, Huber AW, Drescher H, Bersinger NA. Seasonality in human semen quality of smokers and non-smokers: effect of temperature. Asian J Androl. 2004;6(3):243-7.

15.Jurema MW, Vieira AD, Bankowski B, Petrella C, Zhao Y, Wallach E, et al. Effect of ejaculatory abstinence period on the pregnancy rate after intrauterine insemination. Fertil Steril. 2005;84(3):678-81.
16. Cagnacci A, Maxia N, Volpe A. Diurnal variation of semen quality in human males. Hum Reprod.1999;14(1):106-9.

17. Matilsky M, Battino S, Ben-Ami M, Geslevich Y, Eyali $\mathrm{V}$, Shalev E. The effect of ejaculatory frequency on semen characteristics of normozoospermic and oligozoospermic men from an infertile population. Hum Reprod. 1993;8(1):71-3.

18. Elzanaty S, Richthoff J, Malm J, Giwercman A. The impact of epididymal and accessory sex gland function on sperm motility. Hum Reprod. 2002;17(11):2904-11.

19. Gonzales GF, Kortebani G, Mazzolli AB. Leukocytospermia and function of the seminal vesicles on seminal quality. Fertil Steril. 1992;57(5):1058-65.

20. Eddy EM, O’Brien DA. The spermatozoon. In: Knobil E, Neill JD, editors. The physiology of reproduction. $2^{\text {nd }}$ ed. New York: Raven Press; 1994. p. 29-77.

21.Johnson L, Varner DD. Effect of daily spermatozoan production but not age of transit time of spermatozoa through the human epididymis. Biol Reprod. 1988;39(4):812-7.

22. Rolf C, Cooper TG, Yeung CH, Nieschlag E. Antioxidant treatment of patients with asthenozoospermia or moderate oligoasthenozoospermia with high-dose vitamin $\mathrm{C}$ and vitamin $\mathrm{E}$ : a randomized, placebocontrolled, double-blind study. Hum Reprod. 1999;14(4):1028-33.

23. Pasqualotto FF, Sharma RK, Nelson DR, Thomas AJ, Agarwal A. Relationship between oxidative stress, semen characteristics, and clinical diagnosis in men undergoing infertility investigation. Fertil Steril. 2000;73(3):459-64.

24. Alkan I, Simsek F, Haklar G, Kervancioglu E, Ozveri $\mathrm{H}$, Yalcin S, et al. Reactive oxygen species production by the spermatozoa of patients with idiopathic infertility: relationship to seminal plasma antioxidants. J Urol. 1997;157(1):140-3.

25. Pasqualotto FF, Silva CO, Umezu FM, Pasqualotto EB, Salvador M, Agarwal A. Relationship between sexual abstinence period and oxidative stress in infertile men. Fertil Steril. 2005;84 Suppl 1:S458.

26. Tur-Kaspa I, Maor Y, Levran D, Yonish M, Mashiach S, Dor J. How often should infertile men have intercourse to achieve conception? Fertil Steril. 1994;62(2):370-5. 